\title{
A CHARACTERIZATION OF DIFFERENTIAL OPERATORS
}

\section{MARTIN ENGERT}

In this paper we shall give an algebraic characterization of differential-difference operators, i.e. a characterization of those continuous linear operators from $D\left(R^{n}\right)$ in to $L^{2}\left(R^{n}\right)$ which can be constructed by combining partial differential operators, multiplications by locally $L^{2}$ functions, and translation operators. The domain and range of theoperator are relatively unimportant, since we are mainly interested in the form of the operator. But, for convenience, in this paper the symbols $D$ or $\Delta$ will always denote continuous linear operators from $D\left(R^{n}\right)$ into $L^{2}\left(R^{n}\right)$.

For each $b$ in $R^{n}$, define the operator $T(b, D)$ to be $e^{-i b x} D e^{i b x}$, where $e^{i b x}$ and $e^{-i b x}$ denote the operators of multiplication by the exponential functions $e^{i b x}$ and $e^{-i b x}$ respectively. Define $T(D)$ to be the complex linear space of operators spanned by $\left\{T(b, D) \mid b\right.$ in $\left.R^{n}\right\}$. In this paper we shall prove the following theorem:

Let $D$ be a continuous linear operator from $D\left(R^{n}\right)$ into $L^{2}\left(R^{n}\right)$. Then $D$ is a differential-difference operator if and only if $T(D)$ is finite dimensional.

The proof of part of this theorem is immediate. (For notation, see Hörmander [1], particularly pages 9-10.) For suppose

$$
D=\sum_{j=1}^{m} \psi_{j} \tau_{x_{j}} D^{\nu_{j}}
$$

is a differential-difference operator with locally $L^{2}$ coefficients $\psi_{j}$. Then

$$
T(b, D)=\sum_{j} \psi_{j} \exp \left(i b x_{j}\right) \tau_{x_{j}} \nu_{j} ! \sum_{\beta \leq \nu_{j}} \frac{(i b)^{\nu_{j}-\beta} D^{\beta}}{\left(\nu_{j}-\beta\right) ! \beta !}
$$

which is a linear combination of operators in the finite set

$$
\left\{\psi_{j} \tau_{x_{j}} D^{\beta} \mid j=1,2, \cdots, m, \beta \leqq \nu_{j}\right\} .
$$

Thus $T(D)$ is finite dimensional.

Now suppose that $T(D)$ is finite dimensional. In order to show that $D$ is a differential-difference operator, we shall first prove some preliminary lemmas.

Received by the editors July 10, 1966. 
Lemma 1. Suppose $T(b, D)=D+\sum_{j=1}^{m} c_{j}(b) \psi_{j}$ where $\psi_{j}$ denotes the operator of multiplication by the locally $L^{2}$ function $\psi_{j}$ and the functions $\psi_{j}$ are linearly independent. Then $c_{j}(j=1,2, \cdots, m)$ is a complex valued linear function on $R^{n}$.

Proof. We shall show $c_{j}$ is additive and continuous at the origin. This implies that $c_{j}$ is linear.

$$
\begin{aligned}
T\left(b_{1}+b_{2}, D\right) & =\exp \left(-i b_{1} x\right) \exp \left(-i b_{2} x\right) D \exp \left(i b_{2} x\right) \exp \left(i b_{1} x\right) \\
& =\exp \left(-i b_{1} x\right)\left(D+\sum c_{j}\left(b_{2}\right) \psi_{j}\right) \exp \left(i b_{1} x\right) \\
& =D+\sum_{j}\left(c_{j}\left(b_{1}\right)+c_{j}\left(b_{2}\right)\right) \psi_{j} .
\end{aligned}
$$

Also $T\left(b_{1}+b_{2}, D\right)=D+\sum c_{j}\left(b_{1}+b_{2}\right) \psi_{j}$. Thus $c_{j}$ is additive.

Since the functions $\psi_{j}$ are linearly independent, we can choose functions $g_{1}, \cdots, g_{m}$ in $D$ so that $\int \psi_{j} g_{k}=\delta_{j k}$. Thus for a suitably large sphere $S, c_{j}(b)=\int_{S} T(b, D) g_{j}-\int_{S} D g_{j}$. Then $\lim _{b \rightarrow 0} e^{i b x} g_{j}$ converges in $D$ to $g_{j}$, so $\lim _{b \rightarrow 0} D e^{i b x} g_{j}$ converges in $L^{2}$ to $D g_{j}$. Since $e^{i b x \rightarrow 1}$ uniformly on $S$ as $b \rightarrow 0, \int_{S} T(b, D) g_{j}$ converges to $\int_{S} D g_{j}$ as $b \rightarrow 0$. Thus $\lim _{b \rightarrow 0} c_{j}(b)=0$.

Lemma 2. Suppose that for each $b$ in $R^{n}$ and each $f$ in $D$ we have $T(b, D) f=D f$. Then there is a locally $L^{2}$ function $\psi$ so that $D f=\psi f$.

Proof. Let $f$ and $g$ be functions in $D$ such that $g$ is identically one on the support of $f$. Define the function $\psi$ on the set where $g=1$ by $\psi=D g$. We want to show $D f=\psi f$. Let $P_{k}$ be a sequence of trigonometric polynomials such that $g P_{k}$ converges to $f$ in $D$. Since $D$ commutes with multiplication by trigonometric polynomials, $g D P_{k} g$ $=g P_{k} D g \rightarrow g f \psi$ in $L^{2}$. Since we can define $\psi$ in this way on arbitrarily large spheres, we can define $\psi$ on all of $R^{n}$.

LemMa 3. Suppose that the complex linear space spanned by $\{T(b, \Delta)$ $-\Delta \mid b$ in $\left.R^{n}\right\}$ is a finite dimensional space $V$ of differential operators with locally $L^{2}$ coefficients. Then $\Delta$ is also a differential operator with locally $L^{2}$ coefficients.

- Proof. First choose a basis of differential operators for the space $V$ Look at the finite set of coefficient functions that appear in these operators and choose a maximal linearly independent subset $\left\{\psi_{j}\right\}$. Then any operator in $V$ can be written uniquely as $\sum_{\alpha, j} c_{\alpha} \psi_{j} D^{\alpha}$ for some constants $c_{\alpha}^{j}$. In general, there are functions $c_{\alpha}^{j}$ defined on $R^{n}$ so that $T(b, \Delta)-\Delta=\sum c_{\alpha}(b) \psi_{j} D^{\alpha}$. We compute 


$$
\begin{aligned}
T\left(b+b^{\prime}, \Delta\right) & =T(b, T(b, \Delta)) \\
& =T\left(b, \Delta+\sum c_{\alpha}^{j}\left(b^{\prime}\right) \psi_{j} D^{\alpha}\right) \\
& =\Delta+\sum_{\alpha, j} c_{\alpha}^{j}(b) \psi_{j} D^{\alpha}+\sum_{\alpha, j} c_{\alpha}^{j}\left(b^{\prime}\right) \psi_{j} \sum_{\gamma \leq \alpha} \alpha ! \frac{(i b)^{\alpha-\gamma} D^{\gamma}}{(\alpha-\gamma) ! \gamma !} \\
& =\Delta+\sum_{\gamma, j} c_{\gamma}^{j}(b) \psi_{j} D^{\gamma}+\sum_{\alpha, j} c_{\alpha}^{j}\left(b^{\prime}\right) \psi_{j} \sum_{\gamma \leq \alpha} \alpha ! \frac{(i b)^{\alpha-\gamma} D^{\gamma}}{(\alpha-\gamma) ! \gamma !}
\end{aligned}
$$

The coefficient of $\psi_{j} D^{\nu}$ is equal to

$$
\begin{aligned}
\sum_{\alpha \geq \gamma} c_{\alpha}^{j}\left(b^{\prime}\right) \frac{\alpha !}{(\alpha-\gamma) ! \gamma !} & (i b)^{\alpha-\gamma}+c_{\gamma}^{j}(b) \\
= & \sum_{\alpha \geq \gamma} c_{\alpha}^{j}\left(b^{\prime}\right) \frac{\alpha !}{(\alpha-\gamma) ! \gamma !}(i b)^{\alpha-\gamma}+c_{\gamma}^{j}\left(b^{\prime}\right)+c_{\gamma}^{j}(b) .
\end{aligned}
$$

This expression has to be symmetric in $b$ and $b^{\prime}$, giving for each $j$ and $\gamma$ the set of equations

$$
\sum_{\alpha>\gamma} c_{\alpha}^{j}\left(b^{\prime}\right) \frac{\alpha !}{(\alpha-\gamma) ! \gamma !}(i b)^{\alpha-\gamma}=\sum_{\alpha>\gamma} c_{\alpha}^{j}(b) \frac{\alpha !}{(\alpha-\gamma) ! \gamma !}\left(i b^{\prime}\right)^{\alpha-\gamma} .
$$

Now change notation on the right side of this equation by letting $\beta=\alpha-\gamma$. We get

$$
\sum_{\alpha>\gamma} c_{\alpha}^{j}\left(b^{\prime}\right) \frac{\alpha !}{(\alpha-\gamma) ! \gamma !}(i b)^{\alpha-\gamma}=\sum_{\beta>0} c_{\beta+\gamma}^{j}(b) \frac{(\beta+\gamma) !}{\beta ! \gamma !}\left(i b^{\prime}\right)^{\beta} .
$$

The sum on the right is a finite sum, say over $\beta=\beta_{1}, \beta_{2}, \cdots, \beta_{m}$. Choose $b_{1}, \cdots, b_{m}$ so that the matrix $\left[b_{j k}\right]=\left[\left(i b_{k}\right)^{\beta_{j}}\right]$ is invertible. This is equivalent to saying that there exist numbers $B_{\beta, k}$ with the property

$$
\begin{aligned}
\sum_{k=1}^{m}\left(i b_{k}\right)^{\alpha} B_{\beta, k}=1 & \text { if } \quad \alpha=\beta, \\
=0 & \text { if } \quad \alpha \neq \beta .
\end{aligned}
$$

By letting $b^{\prime}=b_{1}, \cdots, b_{m}$ successively in equation (1) above we get for each $\gamma$ and $j$ a set of $m$ equations which we can solve for $((\beta+\gamma) ! / \beta ! \gamma !) c_{\beta+\gamma}^{J}(b)$, finding it equal to

$$
\sum_{k} B_{\beta, k} \sum_{\alpha>\gamma} c_{\alpha}\left(b_{k}\right) \frac{\alpha !}{(\alpha-\gamma) ! \gamma !}(i b)^{\alpha-\gamma}
$$


Thus, if $\beta>0$,

$$
\begin{aligned}
c_{\beta+\gamma}^{j}(b) & =\sum_{\alpha>\gamma} \frac{\beta !}{(\beta+\gamma) !} \sum_{k} B_{\beta, k} c_{\alpha}^{j}\left(b_{k}\right) \frac{\alpha !}{(\alpha-\gamma) !}(i b)^{\alpha-\gamma} \\
& =\sum_{\alpha>\gamma}\left[\sum_{k} B_{\beta, k} c_{\alpha}^{j}\left(b_{k}\right) \frac{\alpha ! \beta !}{(\alpha+\beta) !}\right] \frac{(\alpha+\beta) !(i b)^{\alpha-\gamma}}{(\beta+\gamma) !(\alpha-\gamma) !} .
\end{aligned}
$$

Define $k_{j}(\alpha, \beta)$ to be the quantity within the brackets. Note that $k_{j}(\alpha, \beta)$ is defined only if $\alpha>0$ and $\beta>0$. If we take the special case $\gamma=0$ we get, for $\beta>0$

$$
c_{\beta}^{j}(b)=\sum_{\alpha>0}\left[k_{j}(\alpha, \beta)\right] \frac{(\alpha+\beta) !}{\alpha ! \beta !}(i b)^{\alpha}
$$

which says that $c_{\beta}^{j}$ is a polynomial and that $k_{j}(\alpha, \beta)$ is the coefficient of the monomial $((\alpha+\beta) ! / \alpha ! \beta !)(i b)^{\alpha}$ in the function $c_{\beta}^{J}$.

We want to show that $k_{j}(\alpha, \beta)$ depends only on $j$ and $\alpha+\beta$. We shall show this first in the special case where there are multi-indices $\alpha_{0}, \beta_{0}$, and $\gamma$ such that $\beta=\beta_{0}+\gamma$ and $\alpha=\alpha_{0}-\gamma$. Using equation (2) twice

$$
\begin{aligned}
c_{\beta}^{j}(b) & =\sum_{\alpha>0}\left[k_{j}(\alpha, \beta)\right] \frac{(\alpha+\beta) !}{\alpha ! \beta !}(i b)^{\alpha} \\
& =c_{\beta_{0}+\gamma}^{j}(b)=\sum_{\alpha_{0}>\gamma}\left[k_{j}\left(\alpha_{0}, \beta_{0}\right)\right] \frac{\left(\alpha_{0}+\beta_{0}\right) !}{\left(\beta_{0}+\gamma\right) !\left(\alpha_{0}-\gamma\right) !}(i b)^{\alpha_{0}-\alpha} \\
& =\sum_{\alpha_{0}>\gamma}\left[k_{j}\left(\alpha_{0}, \beta_{0}\right)\right] \frac{(\alpha+\beta) !}{\alpha ! \beta !}(i b)^{\alpha} .
\end{aligned}
$$

So $k_{j}(\alpha, \beta)=k_{j}\left(\alpha_{0}, \beta_{0}\right)$ since they both can be described as the coefficient of $((\alpha+\beta) ! / \alpha ! \beta !)(i b)^{\alpha}$ in $c_{\beta}^{j}$.

From this we can conclude that $k_{j}\left(\alpha_{1}, \beta_{1}\right)=k_{j}\left(\alpha_{2}, \beta_{2}\right)$ whenever we can find a third pair of nonzero multi-indices $\left(\alpha_{3}, \beta_{3}\right)$ so that $\alpha_{1}+\beta_{1}$ $=\alpha_{2}+\beta_{2}=\alpha_{3}+\beta_{3}$, and we can compare $\beta_{3}$ to both $\beta_{1}$ and $\beta_{2}$ in the way we have just done. By simple arguments we can show that if the multi-index $\nu$ has $|\nu| \geqq 3$ and $\alpha_{1}+\beta_{1}=\alpha_{2}+\beta_{2}=\nu$ and if none of the multi-indices $\alpha_{1}, \alpha_{2}, \beta_{1}, \beta_{2}$ is equal to zero, then $k_{j}\left(\alpha_{1}, \beta_{1}\right)=k_{j}\left(\alpha_{2}, \beta_{2}\right)$.

Thus, for $|\nu| \geqq 3$, we can define $k_{j}(\nu)$ as $k_{j}(\alpha, \beta)$ for any $\alpha$ and $\beta$ with $\alpha+\beta=\nu$.

Let $\Delta^{\prime}=\sum_{j} \psi_{j} \sum_{|\nu| \geqq 3} k_{j}(\nu) D^{\nu}$. Then

$$
T\left(b, \Delta^{\prime}\right)-\Delta^{\prime}=\sum_{j} \psi_{j} \sum_{|\nu| \geqq 3} k_{j}(\nu) \sum_{\beta<\nu} \frac{\nu !}{(\nu-\beta) ! \beta !}(i b)^{\nu-\beta} D^{\beta}
$$


By comparing this with equation (3) we can see that there are numbers $k_{j}^{\prime}(\alpha, \beta)$ so that

$$
\begin{aligned}
T(b, & \left.\Delta-\Delta^{\prime}\right)-\left(\Delta-\Delta^{\prime}\right) \\
& =\sum_{j} \psi_{j}\left[\sum_{|\alpha+\beta| \leqq 2} k_{j}^{\prime}(\alpha, \beta) \frac{(\alpha+\beta) !}{\alpha ! \beta !}(i b)^{\alpha} D^{\beta}+c_{0}^{j}(b)\right], \quad \alpha>0 .
\end{aligned}
$$

The right side of this equation contains only partial differential operators of order one and multiplications by functions, so we can define functions $d_{\boldsymbol{k}}^{\jmath}(k=0,1, \cdots, n)$, such that

$$
T\left(b, \Delta-\Delta^{\prime}\right)-\left(\Delta-\Delta^{\prime}\right)=\sum_{j} \psi_{j}\left[d_{0}^{j}(b)+\sum_{k=1}^{n} d_{k}^{j}(b) \frac{\partial}{\partial x_{k}}\right] .
$$

Using the same trick as before:

$$
\begin{aligned}
T\left(b+b^{\prime}, \Delta-\Delta^{\prime}\right) & =T\left(b, \Delta-\Delta^{\prime}+\sum_{j} \psi_{j}\left(d_{0}^{j}\left(b^{\prime}\right)+\sum_{k} d_{k}^{j}\left(b^{\prime}\right) \frac{\partial}{\partial x_{k}}\right)\right. \\
=\Delta-\Delta^{\prime}+ & \sum_{j} \psi_{j}\left[d_{0}^{j}(b)+d_{0}^{j}\left(b^{\prime}\right)\right. \\
& \left.+\sum_{k} d_{k}^{j}\left(b^{\prime}\right) \frac{\partial}{\partial x_{k}}+\sum_{k} d_{k}^{j}(b) \frac{\partial}{\partial x_{k}}+\sum_{k}\left(i b_{k}\right)\left(i b_{k}^{\prime}\right)\right]
\end{aligned}
$$

where $b_{k}$ is the $k$ th coordinate of $b$. This has to be symmetric in $b$ and $b^{\prime}$, so we get

$$
\sum_{k} d_{k}^{j}(b) b_{k}^{\prime}=\sum_{k} d_{k}^{j}\left(b^{\prime}\right) b_{k} .
$$

Let $y_{k}$ be the point in $R^{n}$ that has $k$ th coordinate 1 and all the other coordinates equal to zero. Pick $b^{\prime}$ to be $y_{1}, \cdots, y_{n}$ in succession. We get $d_{\mathbf{k}}^{j}(b)=\sum_{p=1}^{n} d_{p}^{j}\left(y_{k}\right) b_{p}$ and thus, by letting $b=y_{q}, d_{\mathbf{k}}^{j}\left(y_{q}\right)=d_{q}^{j}\left(y_{k}\right)$. Let $\Delta^{\prime \prime}=(1 / 2) \sum_{j} \psi_{j} \sum \sum_{q, k} d_{k}^{j}\left(y_{q}\right)\left(\partial / \partial x_{q}\right)\left(\partial / \partial x_{k}\right)$. Then

$$
\begin{aligned}
T\left(b, \Delta^{\prime \prime}\right)=\Delta^{\prime \prime}+\sum_{j} \psi_{j} & {\left[\frac{1}{2} \sum_{k} d_{k}^{j}\left(y_{q}\right) b_{q} \frac{\partial}{\partial x_{k}}\right.} \\
& \left.+\frac{1}{2} \sum_{q} d_{k}^{j}\left(y_{q}\right) b_{k} \frac{\partial}{\partial x_{q}}+\frac{1}{2} \sum_{k} \sum_{q} d_{k}^{j}\left(y_{q}\right) b_{k} b_{q}\right] \\
=\Delta^{\prime \prime}+\sum_{j} \psi_{j} & {\left[\sum_{k} d_{k}^{j}(b) \frac{\partial}{\partial x_{k}}+\frac{1}{2} \sum_{k} \sum_{q} d_{k}^{j}\left(v_{q}\right) b_{k} b_{q}\right] . }
\end{aligned}
$$

Thus 


$$
\begin{aligned}
T\left(b, \Delta-\Delta^{\prime}-\right. & \left.\Delta^{\prime \prime}\right)-\left(\Delta-\Delta^{\prime}-\Delta^{\prime \prime}\right) \\
& =\sum_{j} \psi_{j}\left[d_{0}^{j}(b)-\frac{1}{2} \sum_{k} \sum_{q} d_{k}^{j}\left(y_{q}\right) b_{k} b_{q}\right] \\
& =\sum_{j} \psi_{j}\left[c_{j}(b)\right] .
\end{aligned}
$$

By Lemma $1, c_{j}$ is a linear function on $R^{n}$, i.e., for each $j$ there are points $P^{j}$ and $Q^{j}$ in $R^{n}$ such that $c_{j}(x)=\left\langle P^{j}, x\right\rangle+i\left\langle Q^{j}, x\right\rangle$. Define $\Delta^{\prime \prime \prime}$ to be $-i \sum_{j} \psi_{j}\left[\left(P_{1}^{j}+i Q_{1}^{j}\right)\left(\partial / \partial x_{1}\right)+\cdots+\left(P_{n}^{j}+i Q_{n}^{j}\right)\left(\partial / \partial x_{n}\right)\right]$. Then $T\left(b, \Delta^{\prime \prime \prime}\right)=\Delta^{\prime \prime \prime}+\sum_{j} \psi_{j}\left[c_{j}(b)\right]$. This implies $T\left(b, \Delta-\Delta^{\prime}-\Delta^{\prime \prime}-\Delta^{\prime \prime \prime}\right)$ $-\left(\Delta-\Delta^{\prime}-\Delta^{\prime \prime}-\Delta^{\prime \prime \prime}\right)=0$. By Lemma $2, \Delta-\Delta^{\prime}-\Delta^{\prime \prime}-\Delta^{\prime \prime \prime}$ is multiplication by a locally $L^{2}$ function. Since $\Delta^{\prime}, \Delta^{\prime \prime}$, and $\Delta^{\prime \prime \prime}$ are all differential operators with locally $L^{2}$ coefficients, so is $\Delta$.

Proof of Theorem. Since $T(D)$ is finite dimensional and since $\left\{T(b, \cdot) \mid b\right.$ in $\left.R^{n}\right\}$ is a commuting family of mappings from $T(D)$ in to itself, we can write $T(D)$ as a direct sum $V^{1} \oplus \cdots \oplus V^{k}$ where each subspace $V^{j}$ is invariant and indecomposable under this family of mappings. Also a basis for $T(D)$ can be found so that on $V^{j}$ the mappings $T(b, \cdot)$ have matrices which are lower triangular with all diagonal elements equal, i.e., of the form

$$
\left[\begin{array}{ccc}
p_{j}(b) & & 0 \\
* & \cdot & \\
& & p_{j}(b)
\end{array}\right]
$$

(see Jacobson [2, p. 134]). It is easily checked that $p_{j}(a+b)$ $=p_{j}(a) p_{j}(b)$, and an argument similar to that in Lemma 1 will show that $p_{j}$ is continuous. Thus $p_{j}$ is an exponential function. We would like to show that $p_{j}$ is bounded. Suppose the contrary: then there is a $b$ in $R^{n}$ so that $\left|p_{j}(b)\right|=\alpha>1$. Let $f$ be in D. Define, for $m$ an integer, $f_{m}=\exp (i m b x) f / \beta^{m}$, where $1<\beta<\alpha$. Then $f_{m}$ converges to zero in $D$. Let $D_{1}^{j}$ be an element of $V^{j}$ such that $T\left(D_{1}^{j}\right)$ is one dimensional. Then $T\left(m b, D_{1}^{j}\right) f=\left(p_{j}(b)\right)^{m} D_{1}^{j} f$ and we have

$$
\left\|D_{1}^{j} f_{m}\right\|_{2}=\left\|\frac{\alpha^{m}}{\beta^{m}} e^{i m b x} D_{1}^{j} f\right\|_{2}=\left|\frac{\alpha}{\beta}\right|^{m}\left\|D_{1}^{j} f\right\|_{2} .
$$

Since $D_{1}^{f}$ is continuous, the left side of this equation must go to zero as $m$ gets large. But the right side grows without bound, so we have a contradiction. Thus $p_{j}$ is a bounded exponential; $p_{j}(x)=\exp \left(i b_{j} x\right)$ for some $b_{j}$ in $R^{n}$. If we let $W^{j}$ denote the space of operators of the form $\tau_{b_{j}} A$ where $A$ is in $V^{j}$ and $\tau_{b_{j}}$ denotes the operator of translation by 
$b_{j}$, then $W^{j}$ is invariant and indecomposable under the family of mappings $\left\{T(b, \cdot) \mid b\right.$ in $\left.R^{n}\right\}$ and there is a basis for $W^{j}$ such that the matrix of $T(b, \cdot)$ on $W^{j}$ is zero above the diagonal and identically one on the diagonal. Applying Lemma 3 to the basis elements of $W^{j}$ in succession, first to the basis element $D_{1}^{j}$ such that $T\left(D_{1}^{j}\right)$ is one dimensional, then to the element $D_{2}^{j}$ such that $T\left(D_{2}^{j}\right)$ is two dimensional, etc., we see that $W^{j}$ consists of differential operators. Thus $V^{j}$ consists of differential-difference operators, which implies that every operator in $T(D)$, including $D$, is a differential-difference operator.

\section{REFERENCES}

1. L. Hörmander, Linear partial differential operators, Academic Press, New York, 1963.

2. N. Jacobson, Lectures in abstract algebra, Vol. II, Van Nostrand, Princeton, N. J., 1953.

University of North Carolina 